\title{
Reducing the costs of peer review
}

Nature Neuroscience is joining a consortium of journals that enables reviews to be transferred from one journal to
another, while allowing authors, referees and editors to control their degree of participation in the system flexibly.

$\mathbf{T}$ he increasing pressure to compete for scarce funding and academic positions motivates authors to submit their papers to the highest ranked journal possible. For this reason, many papers are now being reviewed by several journals before being accepted for publication. The resulting redundancy makes the peer review system inefficient, wasting the time of authors and referees. It also makes life more difficult for editors, who often have difficulty finding reliable referees for all their papers-particularly those that have been shopped around extensively before arriving at the journal.

Monolithic systems, in which a single review process would direct a paper to one of a tiered group of journals, have been proposed to ameliorate these problems. However, such a system would lock authors into a review process that might not be optimal for all papers, leaving the authors without the ability to opt out. In January, a group of editors, supported by the Society for Neuroscience, implemented a more flexible solution that allows voluntary participation by authors, referees and editors, known as the Neuroscience Peer Review Consortium (NPRC; more details at http://nprc.incf.org).

Because we feel that the NPRC provides a valuable service to our community, we are pleased to announce that Nature Neuroscience is now joining the consortium. As a member journal, we will offer authors whose papers are no longer under consideration the opportunity to transfer their reviews when submitting the paper to another consortium journal.

The NPRC reduces the overall reviewing workload of the community by allowing authors to continue the initial review process when their paper moves from one consortium journal to another, once the paper has been rejected or withdrawn from the first journal. This arrangement is similar to the manuscript transfer system that has been available within the Nature family of journals for almost a decade. So far, more than 30 journals, including the Journal of Neuroscience, have become members of the NPRC, representing a substantial fraction of mainstream journals in the field. The full list of journals can be found on the NPRC website.

Like the Nature journals' transfer system, the NPRC system is completely voluntary for authors. Editors at one journal know that a paper was reviewed elsewhere only if the author chooses to inform them. If the reviews from the first journal do not seem likely to facilitate acceptance at another journal, the authors are welcome to send the paper to the second journal directly and have the paper considered as a fresh submission. However, if the author feels that the reviews may be helpful, transferring them can accelerate the editorial process at the second journal, reducing publication delays. Each journal will transfer reviews only once, to ensure that each transfer includes the paper's full transfer history within the NPRC system. That is, once the paper has been considered by a second journal, only that journal can transfer the reviews to a third journal.

Referees also have the option of whether to participate in the transfer system. When they review a paper, they are asked to state whether the editors may release their names along with the review in the event that the paper is transferred to another journal. If a referee declines, that review is passed along to the next journal anonymously. It is most helpful to the recipient journal if the reviews are accompanied by the identities of the referees, so we strongly encourage our referees to participate in the NPRC system whenever possible. All editors within the consortium are committed to maintaining the confidentiality of transferred reviews, just as they would for their own review process, and do not reveal the referees' identities to the authors.

Finally, editors have full discretion in deciding how to use transferred reviews. The receiving editor may choose to accept or reject a paper based on these reviews, without further consideration; to send the paper to some or all of the previous referees for evaluation of the authors' revisions; or to request a fresh set of reviews from new referees.

Only comments to the authors are transferred to the receiving journal. Confidential comments to the editors are not passed along. Thus, to ensure transparency in the review process, both at Nature Neuroscience and at other journals after the paper has been transferred, we encourage referees to include all their concerns about the paper in comments to the authors. The small amount of extra time required to word the comments diplomatically for the authors should be more than counterbalanced by the resulting improvement in the peer review process. Many members of the community have strong views on the issue of confidential comments, which can be found on our blog, Action Potential (http://blogs.nature.com/nn/actionpotential/2007/11/ confidential_comments_your_opi.html).

Referees should use comments to the editors to communicate ethical concerns and for comments that may reveal their identity or other confidential information; for example, to compare the paper to a related paper under consideration that they have also been asked to evaluate. Comments to editors should also be used to indicate whether or not the referee is willing to have his or her name revealed to the receiving editor if the paper is transferred.

The success of the NPRC system will be evaluated at the end of this year. We will also be evaluating our participation on an ongoing basis, so we encourage authors, referees and readers to share their comments with the editors, either on Action Potential (http://blogs.nature.com/nn/actionpotential/nprc) or privately by e-mail to neurosci@natureny.com. 